\title{
A Behavioral Perspective of Virtual Heritage Reconstruction
}

\author{
D.M. Popovici, R. Querrec, C.M. Bogdan, N. Popovici
}

\author{
Dorin-Mircea Popovici, Crenguţa-Mădălina Bodgan, Norina Popovici \\ OVIDIUS University of Constanta \\ 124 B-dul Mamaia, 900527, Constanta, Romania \\ E-mail: \{dmpopovici,cbogdan\}@univ-ovidius.ro,norinapopovici@yahoo.com
}

\section{Ronan Querrec}

Ecole Nationale d'Ingénieurs de Brest

Laboratoire d'Informatique des Systèmes Complexes

Address: 25 rue Claude Chappe, F-29280 Plouzan, France

E-mail: querrec@enib.fr

\begin{abstract}
Our contribution focuses on the behavioral aspects that are currently used in the modeling of the virtual inhabitants of a reconstructed GreekRoman colony in the framework of the TOMIS project. The project aims at promoting culture by the mean of the reconstruction of historical sites together with their virtual societies based on virtual and/or augmented reality technologies. Our efforts are oriented both on 3D modeling of virtual humans, animation of the virtual humans on every-day human activities and, most important, on the spicing these activities with human emotions. To this end, we iterate the most common agent-based architectures used to produce credible behavior of the virtual agents (humans or animals) in situations inspired from the real world, and emphasize their direct applicability both in humans and animal animations in order to obtain complex behavior based on atomic activities. Finally, the paper presents the technological issues related to the used motion capture technology, as source of high-definition human atomic actions, that participates in complex action plans for virtual agents activities.

Keywords: virtual reality, behavior, motion capture, fuzzy-oriented modeling, virtual agent.
\end{abstract}

\section{Introduction}

The 3D historical sites reconstruction is one of the most VR-supported cultural dissemination form. Besides the virtual reconstruction of the old buildings these sites are now animated by means of virtual animals and plants. More important are the virtual humans that perform some individual or collaborative activities. The navigation or exploration of the virtual environment, the triggering of the animation of some mechanisms (cranes, vehicles), and the manipulation of objects are some examples of such activities that users may share with the virtual inhabitants of an ancient site. This is the direction on which we are focused in this paper.

The paper is organised as follows: after a brief presentation of the framework of our effort, in Section 3 we bring into discussion perception, emotion and motivation as main ingredients of a behavioral architecture adopted for our virtual humans. In the sections 4 and 5 we present the application of three behavioral patterns used in the expression of virtual agent action plans. In the last section we discuss the current state of applying motion capture technology in the behavioral modeling of the agent. Finally, we mention some of our directions for the near future and conclusions. 


\section{TOMIS project context}

The major objective of TOMIS project is the development of a multi-sensorial, interactive framework, based on VR/AR technologies that allows the recreation of historical and cultural relics that are inaccessible because of temporal constraints (they existed centuries ago) or geographic constraints (they are placed in submersed areas, great distances or off limits to the general public). To this end we are currently using and developing methodologies and techniques of digital restoration/reconstruction applied on vestiges and artifacts of historical importance, religious and cultural, on the basis of advanced VR/AR technologies. These methodologies and techniques consider also the surrounding elements of flora, fauna and geo-ecology.

The obtained multimodal pilot system will then be evaluated as experimental information technology, both in academic and public setups. To meet all these objectives, the researchdevelopment activities proposed in this project are grouped in three distinct phases: a) geometric modeling of the virtual artefacts, fauna, flora and humans; b) behavioural modeling of fauna, flora and population; and c) virtual environment setup together with interaction devices integration. In the following we focus on the second phase, more specific on population behavioral modelling.

Behavioral modelling focuses on the topology of a group, which allows the specialization of the virtual humanoids in accordance with their competences. In other words, its organization is introduced in terms of roles described as capacities (or tasks) and responsibilities of the group members. From our perspective, we consider this organization to be dynamic, explicit and sensitive to the evolution of the environment in which the virtual humans carry out their activities.

To simulate the behavior of all dinamic components which populate the environment, multiagent systems technology is applied. Each environment element (plant, animal, member of the society or object) has associated an agent that is able to play a scenario. This allows description and compact implementation of a great variety of behaviors, from the simple animation of environmental elements (as fauna, tools or machines used by the people) to the complex interactions of daily human activities.

To this end, we will consider the lowest abstraction level of behavior and primary abilities, attached to society members and elements which populate the environment in which society evolves. On the highest levels of abstraction, we will place the actions (possible collaborative) of society members (in which the user can intervene), which are guided either by collaborative objectives or by individual ones.

\section{Behavioral aspects}

Due to the environment's dynamics, its own physiological and/or emotional state, and its own motivations, the agent is conditioned to evaluate in every moment of its life time, its behavioral resources, and to decide about the action it will select and express as an answer of all these factors. Consequently, the problem of action selection consists in choosing actions necessary for achieving the current goal. Therefore, frequent compromises have to be made, even independent activities have to be combined. In other words, the behavioral selection result have to permit the agent to reach its goals. To this end, the agent credibility is based on a chain of components that realises this stimulus-reaction relationship. These elements as, perception, motivation and emotion are essential to a credible action selection mechanism. 


\subsection{The perception}

The first step in almost every agent's behavioral architecture is to obtain a sensation, which then it transforms into a perception. This perceptual image that the agent creates is dependent of its competencies, goals, knowledges and abilities. Internal or external stimuli, as active entities, produce a reaction from an excitable organism [1].

The information processing may be made in different manners. Tu and Terzopoulos use a neural architecture that maps the sensorial information in neural inputs of their fishes [2]. They implement a visual and temperature sensor and use a focalisation subsystem that elimines any non-important sensorial information. In [3], the visual sensor is based on computer vision algorithms, being inspired from primates visual aparatus. Different perceptive systems may combine by means of fusion percepts to obtains concepts of a higher level of abstraction, prooving this way a great dependence of the agent on its environment [4]. An active perceptual system can demand that some actions be realized in order to extract supplementary information from environment [5]. This way, the perception is guided by behavioral needs, so by actions that needs to be realized.

After the perception takes place in the agent's aura [6], this perceptual information is passed through the sensorial quality filter [7], and produces a separation between the environment's state and its perception by the agent.

\subsection{The motivation}

The motivational states are agents' emotional states that determine itself to react somehow by a specific action. Bolles' and Fanselow's [8] model explores the relation between motivational and emotional states, in particular between fear and pain, as emotions that interrupt the brain in order to impose some kind of neccessity or action. For example, if an agent is hungry, then its brains will redirect all its cognitive ressources in order to make him to search the food. This will favorise the satisfaction of its hungry.

Wright uses the motivator term, for an information subclass, such as desires, goals and intentions, which have the potential to trigger an internal or external agent's action [9]. For Aylett, motivation is a long term goal, an emotional or motor state, depending on the domain, and represents the central element of actions' planning algorithm [10]. This way, the motivational states have a major impact in the emotional and decisional processes, so in the behavioral one.

\subsection{The emotion}

In the theories of emotion, the individual realises a cognitive evaluation of its current state relative to a desired non-riched state in the moment of the evaluation. Reilly [11] proposes as fear model "the likelihood of failing to achieve the current goal" multiplied with "the importance of not failing", while LeDoux [12] affirms that emotion may action at a level much lower than the cognitive one, since the animals may feel the emotions without aware of the cause.

Velasquez [13] uses emotional memories in order to permit agents to chose their actions according to their emotional state. Doing so, the decisional process is directed in an emotiondependent manner. Gratch and Marsella's [14] agents credibility is based on the obtained emotion, on the evaluation of the relations between the events that appear in a given context and the agents goals and plans. El-Nasr [15] also places the emotion in the center of its architecture. After computing the event's desirability, he uses a version of Ortony's model [16] to define, on Fuzzy logic basis, the resulting emotion against current situation and context.

These are our reasons to consider attention, emotion, and motivation as inhibited/exciting factors of the behavioral answer of the agent. 


\section{Agent actions}

The environment that we model is an ancient Greek-Roman colony situated on the Black Sea coast. Here, the main activities of the population take place around the Tomis colony harbour site where we find different social classes of virtual humans, from sailors and commerciants to simple individuals who are looking to buy some market products. Of course, the place is also spiced by the existence of animals or tehnical devices used in market/harbour maneuvers, as ships, cranes, wheelebarrows, etc. All these elements are modeled by the means of virtual agents, as they are defined in [6]. There the agent is considered as a 5-uple:

$$
A g=(F, K, \operatorname{Rec}, E f f, \text { Dec })
$$

where $F$ is the set of the agent's attribute shapes, $K$ represents the agent's knowledge, Rec the set of receptors, Eff the set of this effectors and Dec is the decisional module. While the knowledge is considered as a set of valued concepts and the decidor is a FCM-based module, the receptors and effectors are active entities responsible with the actualisation of sensorial and motors concepts in the decider (fig. 1). Moreover, the effectors, which encapsulate the agent changes as imperative methods in containers of activity, are activated/deactivated at the decider level where the agent realises the action selection.

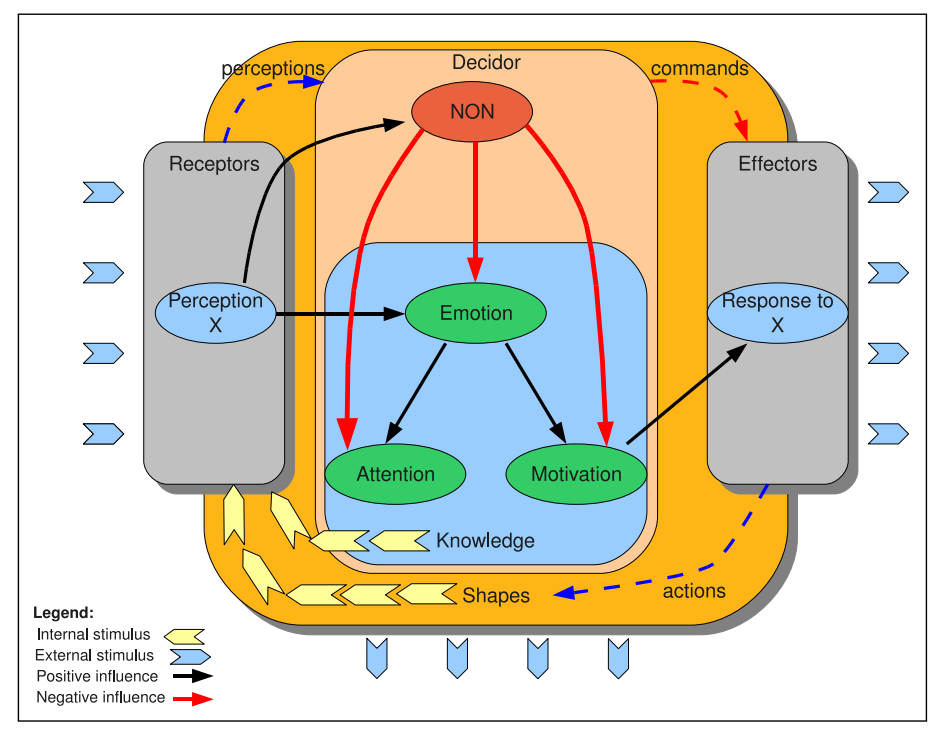

Figure 1: Affective influences in the virtual agent architecture [6]

Obtaining a perception at the level of a Rec produces the appearance of an emotion that triggers the increasing of the attention in what it concerns future perceptions and also the increasing of the motivation to displaying an answer at this perception, by the means of agent effectors. In the same time, a high rate of non-motivation triggered by another perception (even internal to the agent) may produce a strong inhibition of the perception and so of the emotion and answer.

The problem is that even we can determine which will be the agent's a answer to a specific perception, we cannot determine its answer in a complex situation, as in the real environment case. And this, because of the multitude of the obtained perceptions. 


\section{Action selection}

There are two levels toward which we oriented the behavioral modeling in the Tomis colony. The simplest level does not suppose semantic abstraction and is approached by means of behavioral animation. This is the level where the simplest behaviors are implemented, and called atomical actions of the virtual agents. This is the case for seagulls, cranes, ships and other elements animation. In order to filter even this kind of animation we implemented a level of behavior filter that depends only on the distance between the agent and the viewer.

If an element may support different behavioral animations, we chose to implement a finite state machine selection mechanism in order to be able to switch from one state (that corresponds to one behavioral animation) to another. This is the case for "hitting with hammer", "draw/push a dustcart", etc. This means that it is possible that an action depends on the existence of some resources in the very near vecinity of the agent.

These lower abstracted agent capabilities are then used at the next level of abstraction, in the realisation of highly complex behaviors. The high level one is represented by actions as: Load/Unload ship, Taking goods, Guard a zone, Buy a market product, etc. Here, the action may be so complex that she needs a plan of realisation, based on simpler actions.

By using the behavioral patterns, FOF (firstOf), ALL (all) and SEQ (sequence), as defined in [6], we are able to express sequential (SEQ) actions, as well as collaborative (ALL) or even concurrential (FOF) ones. Let us give some example of such actions.

We identified two types of virtual humans: one that express individual behavior, and that plays roles as Porter, Buyer, Merchant, Publican, Teamster; and another that express group behavior, and that plays roles as GroupMember, Soldier / Guardian (despite the fact that he behaves alone, he is part of the Group), as well as Rower, Pairs, Captain. At the level of group behaviors we adopted a boid-oriented solution [17], either by introducing a leader inside the ierarchies (as for Soldier / Rower ...), either by letting the virtual agents to organise themselves (as for GroupMember) without having neccessary a leader.

No matter what is the virtual environment state, the planning of one or several virtual humans' behavior consist mainly in movement allocation by the means of their effectors.

To exemplify an action plan, we adopted a goal oriented approach and consider the high level action "Transport a thing". We chosed to decompose this complex activity into simpler actions, until we reach the atomic actions level for each task. This gives us the following actions sequences:

Transport $<\mathrm{T}>$ From $<\mathrm{S}>\mathrm{To}<\mathrm{D}>$
- SearchFor $<\mathrm{S}>$
- Reach $<\mathrm{S}>$
- SearchFor $<\mathrm{T}>$
- Reach $<\mathrm{T}>$
- Take $<\mathrm{T}>$
- SearchFor $<\mathrm{D}>$
- Reach $<\mathrm{D}>$
- Release $<\mathrm{T}>$
- Explore $<>$

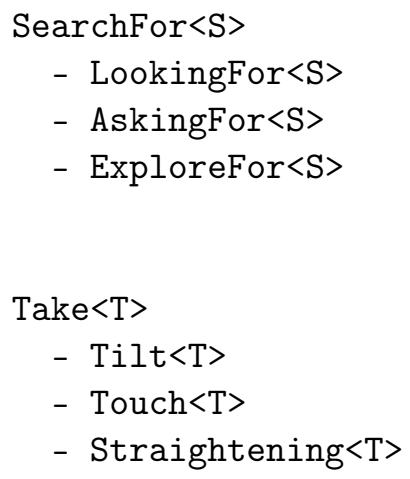

SearchFor $\langle\mathrm{S}>$ means that the agent may explore the environment by looking arround for $\langle\mathrm{S}\rangle$ and if he/she meets someone else it may ask for $\langle\mathrm{S}\rangle$. This is an example of using FOF operator: SearchFor $<\mathrm{T}>=\mathrm{FOF}$ (LookingFor $<\mathrm{T}>$, AskingFor $<\mathrm{T}>$, ExploreFor $<\mathrm{T}>$ ).

Once the information is obtained, he/she triggers to Reach $\langle\mathrm{S}\rangle$. Reach $\langle\mathrm{S}\rangle$ is considered that maybe realized just by relatively simple movements and obstacle avoidance. 
Take $<\mathrm{T}>$ and Release $<\mathrm{T}>$ are two complex opposite actions because the resources they use (in order to take a big object we need both hands). In other words:

Take $<\mathrm{T}>=\mathrm{SEQ}$ (Tilt $<\mathrm{T}>$, Touch $<\mathrm{T}>$, Straightening $<\mathrm{T}>$ )

Release $<\mathrm{T}>=\mathrm{SEQ}(\mathrm{Tilt}<\mathrm{T}>$, Free $<\mathrm{T}>$, Straightening $<\mathrm{T}>$ )

For prooving the ALL operator we will change the action in the market place. Here we suppose to have a virtual human that have to buy several products without knowing exactly if he/she will find the products in the market and where this products are placed. So, for example, let us suppose that the human want to buy some perfume $(\mathrm{PE})$, one $\operatorname{crater}^{1}(\mathrm{CR})$, and some wine (WI). To express this, we use the ALL operator as follows:

Buy $<\mathrm{PE}, \mathrm{CR}, \mathrm{WI}>=\mathrm{ALL}($ Buy $<\mathrm{PE}>$, Buy $<\mathrm{CR}>, \mathrm{Buy}<\mathrm{WI}>)$

For a "buy" activity that is supposed to give the agent the market product $\langle\mathrm{T}\rangle$ after the payment is made, we may consider a sequence like Buy $<\mathrm{T}>=\mathrm{SEQ}$ ( $\operatorname{Search}<\mathrm{T}>$, Reach $<\mathrm{T}>$, Take $<\mathrm{T}>$ ). But when the agent has to buy more than one product, this sequentiality is broken by the inpredictibility of the existence and the topology of the market products. So, we chosed to express the "buy" action as follows:

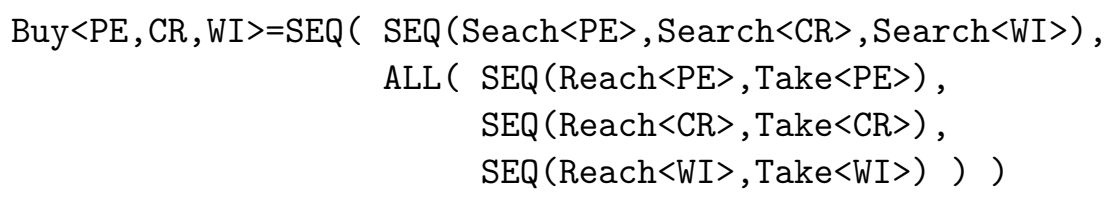

This means that our agent will first evaluate the environment and then he will proceed to achieve the market products according to their accesibility.

Using these behavioral patterns we are also able to decide the failure of actions. To this end, we use time restricted version of operators. So, if the agent fails to complete an action in the corresponding time interval, then the agent will drop the action and will evaluate if it is coherent to continue the current action plan or to change it.

The agent motivation and the resources accesible to the agent are essential. The agent action have to have sense, i.e. to be accorded to its internal state, its perceptions, knowledge about its environment and its capabilities; the agent have to equilibrate its actions between an opportunist behavior and the goal oriented one.

Last but not least, the agent may choose to make a compromise between multiple concurrent behaviors for satisfy a maximum number of goals in the same time.

\section{Some techniques}

As we have already said, the behaviors were implemented using very different techniques, starting from behavioral animation and ending with motion capture solutions.

Behavioral animations were obtained either as morphing shapes (as is the case of seagulls), either directly by procedural animation (as is the case for plants or simple artifacts, as cranes, etc). Nevertheless, a level of behavior was implemented in order to filter the displayed behaviors according to the user's field of view.

Human natural gestures and actions are obtained using motion capture technology. Here challenge was to apply the real-time captured animation to the existed models in order to enhance these 3D models with animation information. Once the animation fitted, we stored the new

\footnotetext{
${ }^{1}$ Crater - small container for mixing wine and water.
} 
models in MD5 or SMD file formats for later use. In the phase of the project we used software tools as 3DS Max [18] and Blender [19] for 3D modeling and Arena software [20] for real-time motion capture. In the figure 2 are presented some examples of the human natural captured actions that are applied at the level of virtual humans in our project.
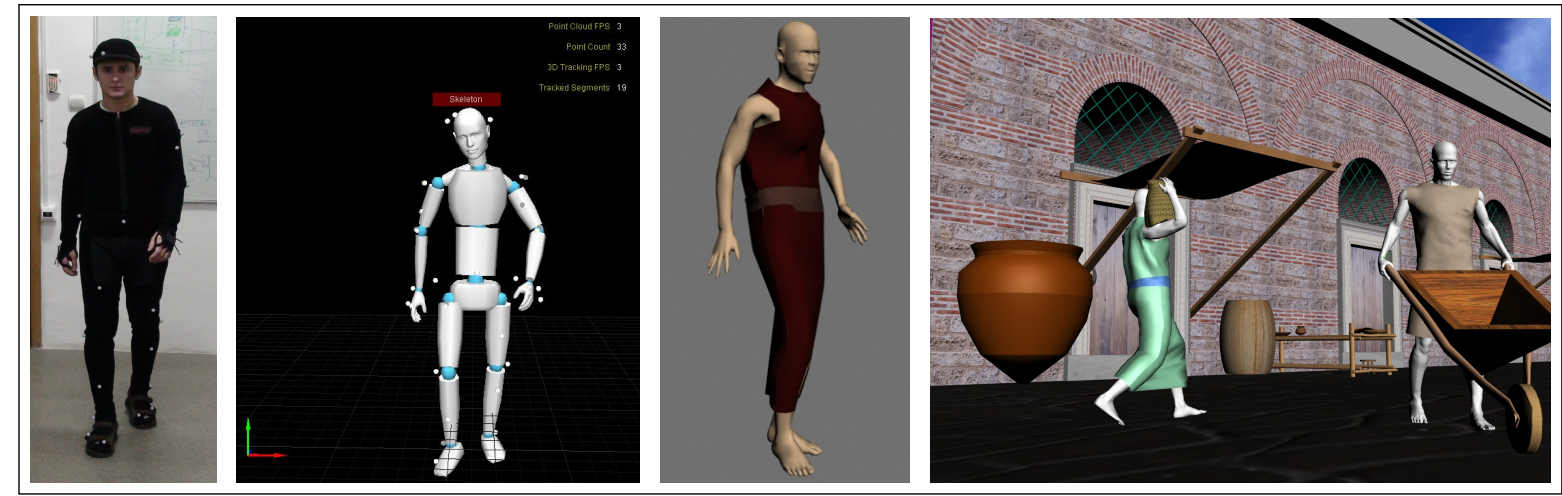

Figure 2: Simple walk action for the real actor, the skeleton, the virtual human, and some other actions applied to virtual humans in TOMIS project.

\section{Conclusions and Future Works}

The credibility of the user experience in the reconstructed environment is augmented by the behavior realism of the virtual humans that the user meets. To this end, we invoked in our solution both emotional aspects and technical ones, and explain how they integrates in the adopted agent architecture. Once the action selection mechanism is tested the mix of several captured motion for complex actions will provide the expected realism to the virtual humans.

\section{Acknowledgements}

Our work is funded by the National Centre of Programs Management through TOMIS project (PN II: 11-041/2007). We also thank to our enthousiastic volunteer members of CERVA team Alexandru Dinca, Manuel Galiu, Ciprian Ilie, Daniela Panait and Mihai Polceanu. For more information please visit our web site http:/www.cerva.ro.

\section{Bibliography}

[1] N. Richard, Description de comportements d'agents autonomes évoluant dans des mondes virtuels, PhD thesis, Ecole Nationale Supérieure des Télécommunications, Paris, 2001.

[2] X. Tu, D. Terzopoulos, Artificial fishes: Physics, locomotion, perception, behavior, Computer Graphics, 28(Annual Conference Series):43-50, 1994.

[3] J.J. Kuffner, Autonomous Agents for Real-Time Animation, PhD thesis, Department of Computer Science of Standford University, 1999.

[4] R.C. Arkin,Behavior-based robotics, MIT Press, 1998.

[5] D. Thalmann, H. Noser, Z. Huang Autonomous virtual actors based on virtual sensors, Lecture Notes in Artificial Intelligence (LNCS), 1997. 
[6] D.M.Popovici, Modelling the space in virtual universes, $\mathrm{PhD}$ thesis, Politehnica University of Bucharest, Romania, 2004.

[7] D. Isla, B. Blumberg, New challenges for character-based ai for games, In AAAI SpringSymposium on AI and Interactive Entertainment, 2002.

[8] R.C. Bolles, M.S. Fanselow, A perceptual defensive recuperative model of fear and pain, Behavioral and Brain Sciences, 3:291-301, 1980.

[9] I. Wright, Emotional Agents, PhD thesis, University of Birmingham, 1997.

[10] R. Aylett, A. Coddington, G. Petley, Agent-based continuous planning, In Proceedings of the 19-th Workshop of the UK Planning and Scheduling Special Interest Group (PLANSIG 2000), 2000 .

[11] W.S.N. Reilly, Believable Social and Emotional Agents, PhD thesis, Carnegie Mellon Univ, 1996.

[12] J. LeDoux, The Emotional Brain, New York: Simon and Schuster, 1996.

[13] J.Velasquez, When robots weep: Emotional memories and decision-making, In Proceedings of the Fifteenth National Conference on Artificial Intelligence, Madison, Wisconsin, 1998. AAAI Press.

[14] J. Gratch, S. Marsella, Tears and fears: modeling emotions and emotional behaviors in synthetic agents, In Jorg P. Muller, Elisabeth Andre, Sandip Sen, and Claude Frasson, editors, Proceedings of the Fifth International Conference on Autonomous Agents, pages 278-285, Montreal, Canada, 2001. ACM Press.

[15] M.S. El-Nasr, J. Yen, T.R. Ioerger, Flame-fuzzy logic adaptive model of emotions, Autonomous Agents and Multi-Agent Systems, 3(3):219-257, 2000.

[16] A. Ortony, G.L. Clore, A. Collins, The Cognitive Structure of Emotions, Cambridge University Press, New York, 1988.

[17] C.W. Reynolds, Flocks, herds and schools: a distributed behavioral model, Computer Graphics (SIGGRAPH'87), 21(4):25-34, 1987.

[18] http://www.autodesk.com

[19] http://www.blender.org

[20] http://www.naturalpoint.com/

[21] C. Rose, B. Bodenheimer, M.F. Cohen, Verbs and Adverbs: Multidimensional Motion Interpolation Using Radial basis Functions, IEEE Computer Graphics and Applications, 18:3240,1998 . 